\title{
Preparation and evaluation of chitosan based thermoreversible gels for intraperitoneal delivery of 5-fluorouracil (5-FU)
}

BHAVESH P. DEPANI

ANUJA A. NAIK

HEMA A. NAIR*

Department of Pharmaceutics, Bombay College of Pharmacy, Kalina, Santacruz (E) Mumbai, India

\begin{abstract}
Sterile thermoreversibly gelling systems based on chitosan-glycerol phosphate were developed for intraperitoneal delivery of the antineoplastic agent 5-FU. The formulation was evaluated for gelling characteristics and in vitro drug release. Drug free gels were evaluated for in vitro cytotoxicity in L-929 mouse fibroblast cells. Drug loaded gels were subjected to acute toxicity studies in Swiss albino mice via intraperitoneal route and efficacy studies via intratumoral injections in subcutaneous colon carcinoma bearing BALB/c mice. The formulations gelled reversibly in $8 \mathrm{~min}$ at $37^{\circ} \mathrm{C}$ and provided prolonged release of the drug. Drug free systems showed dose dependent cytotoxicity in fibroblast cells, while in vivo studies revealed a 2.8 -fold increase in $L D_{50}$ of 5-FU administered intraperitoneally as the developed system. Tumor volume measurements showed comparable efficacy of 5-FU administered as gel and commercial injection with a greatly improved safety profile of the former as adjudged from mortality and body weight measurements.
\end{abstract}

Keywords: chitosan, glycerophosphate, thermoreversible gels, intraperitoneal, 5-fluorouracil

The intravenous route remains the preferred route for anticancer therapy. A less explored, albeit useful, portal for administration is into the peritoneal cavity via intraperitoneal injection. The fundamental goal of intraperitoneal administration of antineoplastic agents is to increase the exposure of cancer cells within the peritoneal cavity to the drug while minimizing potential toxic effects on internal organs. This may provide significant advantages in the treatment of cancers of peritoneal organs. 5-FU is an anti-neoplastic drug used in the palliative treatment of cancers of the ovary, gastrointestinal tract, breast, respiratory tract, etc. (1). 5-FU has high activity in the treatment of drug resistant colon cancer. It has been most widely used for the treatment of breast cancers, ovarian cancer and it is also used in combination with other anti-cancer drugs like leucovorin (2). A number of marketed injectable formulations of 5-FU are available as solu-

*Correspondence; e-mail: hemastudent@gmail.com 
B. P. Depani et al:: Preparation and evaluation of chitosan based thermoreversible gels for intraperitoneal delivery of 5-fluorouracil (5-FU), Acta Pharm. 63 (2013) 479-491.

tions for intravenous administration and as topical creams and ointments for the treatment of actinic keratoses (3).

The antineoplastic activity of 5-FU is exerted by blocking the methylation reaction of deoxyuridylic acid to thymidylic acid leading to thymine deficiency and consecutively interference with the synthesis of DNA and, to a lesser extent, inhibition of the formation of RNA, unbalanced growth and death of the cell. The effects of DNA and RNA deprivation are most marked on those cells which grow more rapidly and which take up 5-FU at a more rapid rate. Hence, intravenous antineoplastic therapy using 5-FU is associated with several adverse effects on cardiac, hematologic, neurological and gastrointestinal systems (4). Intraperitoneal 5-FU chemotherapy is reported to increase exposure to higher drug concentrations for a longer period of time in tumors of the peritoneal cavity while reducing systemic effects. Improved cytotoxicity is attributed to free diffusion of the drug from the peritoneal surface into the tumor (5). This is of special relevance since 5-FU is widely used for the treatment of cancers of organs in the peritoneal cavity, including abdominal and ovarian cancers.

Chitosan is the deacetylated derivative of chitin, a natural component of shrimp and crab shells. It is a biocompatible, cationic polymer, soluble in aqueous media at $\mathrm{pH}$ $<6.2$ (depending upon the degree of deacetylation) (6). Basification of chitosan solutions above this $\mathrm{pH}$ leads to precipitation. $\mathrm{pH}$-gelling, cationic chitosan solutions can be transformed into thermally sensitive, $\mathrm{pH}$-dependent, gel-forming systems by the addition of polyol salts such as glycerolphosphate (GP) $(6,7)$. GP is an organic compound naturally found in the body, which is usually used as a source of phosphate in the treatment of imbalance of phosphate metabolism. Its venal administration has been approved by FDA (8). In the present study, GP represents the disodium salt of $\beta$-glycerophosphate. Chitosan-glycerolphosphate (C-GP) systems possess a neutral $\mathrm{pH}$, remain liquid at or below room temperature, and form monolithic gels at body temperature (9). Hence, they offer a unique advantage in drug therapy in that they are injectable fluids at room temperature and would be converted in vivo to compact depots for prolonged drug release.

The present project was aimed at formulation of a chitosan based thermoreversible gel for intraperitoneal administration of 5-FU. We hypothesized that the formulation would gel post injection and provide high local concentrations of 5-FU in a sustained manner, thereby increasing efficacy, reducing systemic toxicity and offering the possibility of reducing frequent drug administration. The gel was evaluated for in vitro toxicity in mouse L-929 fibroblasts and for in vivo toxicity and efficacy in suitable mice strains.

\section{EXPERIMENTAL}

\section{Materials}

Chitosan (degree of deactylation $\sim 89 \%$ and molecular weight $\sim 73000 \mathrm{Da}$ ) was supplied by the Central Institute of Fishery Technology, Cochin, India. GP was purchased from the Central Drug House, Delhi, India. 5-FU was a gift from Naprod Life Sciences Ltd. Mumbai, India. Dulbecco's Modified Eagle's Medium (DMEM) was procured from Himedia, Mumbai, India, Foetal calf serum, 3-(4,5-dimethylthiazol-2-yl)-2,5-dimethyl te- 
B. P. Depani et al:: Preparation and evaluation of chitosan based thermoreversible gels for intraperitoneal delivery of 5-fluorouracil (5-FU), Acta Pharm. 63 (2013) 479-491.

trazolium bromide (MTT), dimethyl sulfoxide (DMSO) and trypsin were purchased from Sigma-Aldrich, USA. L-929 mouse fibroblast cells were procured from NCCS, Pune, India.

\section{Preparation of sterile chitosan-GP-5-FU (C-GP-5FU) gels}

Sterile formulations were prepared by aseptic processing of presterilized components. 5-FU was sterilized by $\gamma$-radiation (2.5 Mrad) at BRIT, Mumbai, India. A solution of chitosan $(0.1 \mathrm{~g})$ in $0.1 \mathrm{~mol} \mathrm{~L}-1 \mathrm{HCl}(4 \mathrm{~mL})$ was subjected to autoclaving at $121{ }^{\circ} \mathrm{C}$ for 15 $\mathrm{min}$ at $103.4 \mathrm{kPa}$. To the sterilized chitosan solution, presterilized 5-FU (62.5 mg) was added under a laminar flow hood and dissolved. GP was weighed, dissolved in distilled water, and sterilized by passage through a $0.22 \mu \mathrm{m}$ membrane syringe filter (Millipore) under the laminar flow hood to make $50 \%(\mathrm{~m} / \mathrm{V})$ GP solutions. Both chitosan and GP solutions were cooled to $15{ }^{\circ} \mathrm{C}$. $1 \mathrm{~mL}$ of GP solution was added dropwise into the cold chitosan solution under aseptic conditions with continuous mixing using a cyclomixer.

The above procedure resulted in the preparation of a C-GP-5FU system containing $2 \%(\mathrm{~m} / \mathrm{V})$ chitosan, $10 \%(\mathrm{~m} / \mathrm{V})$ GP and $12.5 \mathrm{mg} \mathrm{mL}^{-1} 5$-FU. Drug free mixtures were prepared in a similar manner, except that addition of 5-FU into the chitosan solution was omitted.

\section{Evaluation of C-GP-5FU gel}

Gelation temperature, time and $p H$ (10). - The gelling temperature was measured by immersing the test tubes containing sols in a thermostated water bath and increasing the temperature gradually from 15 to $40{ }^{\circ} \mathrm{C}$ at a rate of $0.5{ }^{\circ} \mathrm{C} \mathrm{min}-1$. The temperature was maintained stable for $10 \mathrm{~min}$ at $15,25,37$ and $40{ }^{\circ} \mathrm{C}$. The tubes were inverted at frequent intervals until movement of the meniscus of the sol on tilting of the tube was arrested.

Fig. 1. Appearance of gel at different temperatures a) 15 , b) 25 , c) 37 , d) $40{ }^{\circ} \mathrm{C}$.
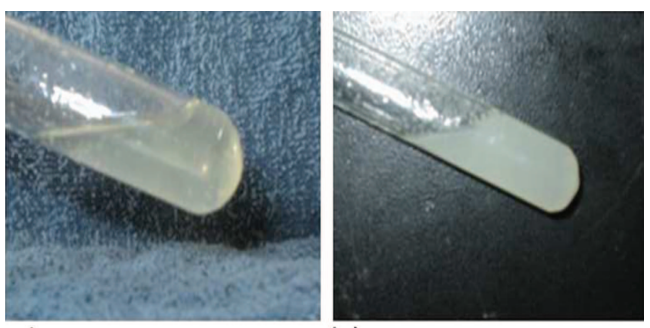

a)

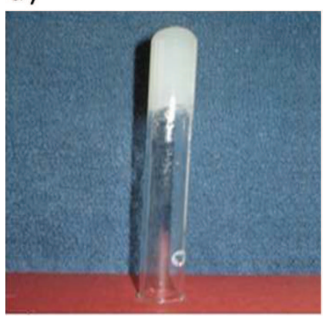

c)

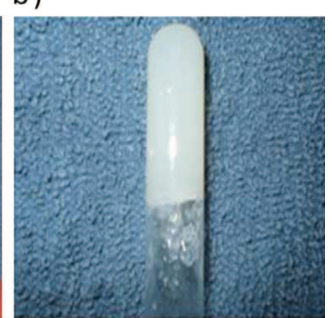

d) 
B. P. Depani et al:: Preparation and evaluation of chitosan based thermoreversible gels for intraperitoneal delivery of 5-fluorouracil (5-FU), Acta Pharm. 63 (2013) 479-491.

Gelation time was measured as the time required to stop the flow of gel at $37{ }^{\circ} \mathrm{C}$ on immersing the solutions in a thermostatic water bath. Pictures of sol-to-gel transformation were recorded with the help of a digital camera. $\mathrm{pH}$ of the gel was recorded at room temperature using a standardized $\mathrm{pH}$ meter.

In vitro drug release. - In vitro drug release from the formulation gelled at $37^{\circ} \mathrm{C}$ was determined in duplicates using a USP dissolution apparatus 4 - flow through cell (Sotax CE-1 with cell 3252). A ruby bead was placed in the lower half of the cell, followed by loading of glass beads $1 \mathrm{~mm}$ in diameter $(1 \mathrm{~g})$ into the release compartment of the apparatus. The upper half of the cell with a capacity of $5 \mathrm{~mL}$ was fitted on the lower one. C-GP-5FU gel ( $1 \mathrm{~g})$ was then gently placed over the glass beads. Stainless steel sieves were placed above the upper half of the cell, followed by a $0.2-\mu \mathrm{m}$ membrane filter. The whole assembly was covered by the adapter containing output tubing, placed in a water bath maintained at $37^{\circ} \mathrm{C}$ and allowed to equilibrate for 15 min prior to commencing the flow of the release medium (Fig. 2).

In operation, the release medium (distilled water) was pumped at a rate of $10 \mathrm{~mL}$ $\mathrm{h}^{-1}$ and was delivered past the sample in the cell. Fresh medium was continuously pumped and the eluate was collected after $0.5,1,2,3,4,5,6,8,10$ and $12 \mathrm{~h}$. The medium from the system was collected as the entire outflow over the sampling interval. Aliquots were diluted appropriately and the 5-FU released over a period of $12 \mathrm{~h}$ was quantified by UV spectrophotometric measurement at $266 \mathrm{~nm}$. Release profile was plotted as cumulative percent released vs. time.

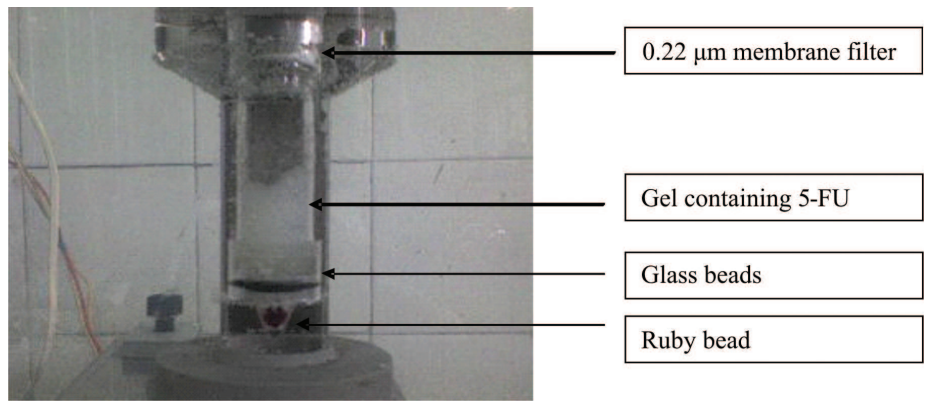

Fig. 2. Flow through a cell containing C-GP-5FU gel.

\section{In vitro cell line cytotoxicity studies}

L-929 mouse fibroblast cells were used to assess the cytotoxicity of drug free C-GP systems. Cells were subcultured in DMEM containing $10 \%$ fetal calf serum and maintained in a humidified atmosphere $\left(5 \% \mathrm{CO}_{2} / 95 \% \mathrm{O}_{2}\right)$ until they were at least $75-80 \%$ confluent, with good morphology. $1 \mathrm{~mL}$ trypsin was added to disaggregate the cells. Culture vessels were incubated at $37^{\circ} \mathrm{C}$ for two minutes, after which cell detachment was monitored under the microscope. Once the cells were detached, $1 \mathrm{~mL}$ DMEM was added to dilute the trypsin and to disperse the cells. The cells were transferred to a ster- 
B. P. Depani et al:: Preparation and evaluation of chitosan based thermoreversible gels for intraperitoneal delivery of 5-fluorouracil (5-FU), Acta Pharm. 63 (2013) 479-491.

ile conical centrifuge tube and centrifuged at $900 \mathrm{rpm}$ for $2 \mathrm{~min}$ followed by dilution with culture medium to attain a cell density of $2 \times 10^{-5}$ cells $\mathrm{mL}^{-1}$. Quantification was achieved using hemocytometry.

A 96-well plate was seeded with $100 \mu \mathrm{L}$ of the above cell suspension, resulting in 20,000 cells in each well. Six cells were reserved as blank, which contained only $100 \mu \mathrm{L}$ of medium. The plates were incubated at $37^{\circ} \mathrm{C}$ in a humidified atmosphere $\left(5 \% \mathrm{CO}_{2} / 95 \%\right.$ $\mathrm{O}_{2}$ ) for $24 \mathrm{~h}$.

After $24 \mathrm{~h}$, different amounts of sterile drug free C-GP systems per well (10, 25, 50, $100 \mu \mathrm{L}$ ) were added to six wells each. In wells with 10,25 and $50 \mu \mathrm{L}$, of the test formulation, a further corresponding amount of DMEM was added to ensure a total volume of $100 \mu \mathrm{L}$ before addition of the test formulation. Six wells containing only cells and medium served as the control. At the end of an incubation period of $24 \mathrm{~h}$, the supernatant medium and test material were removed from all the wells using a fine gauge needle and replaced with $100 \mu \mathrm{L}$ of a $2 \mathrm{mg} / \mathrm{mL}$ MTT solution in phosphate buffered saline A. The plates were wrapped in aluminium foil to protect them from light and incubated for $4 \mathrm{~h}$ in an atmosphere of $5 \% \mathrm{CO}_{2}$.

At the end of $4 \mathrm{~h}$, the supernatant MTT solution was removed using a fine gauge needle and $100 \mu \mathrm{L}$ of DMSO solution was added to dissolve the formazan crystals. Absorbance was recorded at $540 \mathrm{~nm}$ on an ELISA plate reader.

Taking the mean absorbance of the control wells as corresponding to $100 \%$ proliferation, percent proliferation for all the treated wells was estimated. Data was analyzed for statistical significance using ANOVA and Bonferroni's multiple comparison tests.

\section{Toxicity studies}

Acute toxicity studies: Determination of $\mathrm{LD}_{50}$ - In vivo toxicity of the formulation was assessed in Swiss albino mice. The studies were approved by the institutional ethics committee of the Bombay College of Pharmacy, Kalina, Mumbai, India, for animal experiments protocol no. 30/2007. $L D_{50}$ was determined by the up-and-down method as described in OECD guidelines (11) using the Statistical Program (AOT425StatPgm).

The animals were fasted for $24 \mathrm{~h}$ prior to dosing. C-GP-5FU formulation was administered in a single dose by intraperitoneal route with a 22 gauge needle. Each animal

Table I. Observations of animal mass and mortality during toxicity studies

\begin{tabular}{ccccc}
\hline Animal & $\begin{array}{c}\text { Dose of 5-FU admi- } \\
\text { nistered (mg kg-1 } \\
\text { body mass) }\end{array}$ & $\begin{array}{c}\text { Mass of animal } \\
\text { at the start }(\mathrm{g})\end{array}$ & $\begin{array}{c}\text { Mass of animal at } \\
\text { the end of } 48 \mathrm{~h} \mathrm{(g)}\end{array}$ & Mortality \\
\hline 1 & 100 & 32.0 & 29.3 & survived \\
2 & 550 & 27.7 & 23.6 & dead \\
3 & 175 & 32.4 & 29.4 & survived \\
4 & 550 & 34.0 & 26.8 & dead \\
5 & 175 & 28.6 & 26.7 & survived \\
6 & 550 & 27.0 & 20.8 & dead \\
\hline
\end{tabular}


B. P. Depani et al.: Preparation and evaluation of chitosan based thermoreversible gels for intraperitoneal delivery of 5-fluorouracil (5-FU), Acta Pharm. 63 (2013) 479-491.

was dosed in sequence, usually at $48 \mathrm{~h}$ intervals. The first animal was dosed at the best preliminary estimate of $L D_{50}$ of 5 -FU as found in the literature $\left(100 \mathrm{mg} \mathrm{kg}^{-1}\right)$. The subsequent animal received a higher dose (if the earlier animal survived) or a lower dose (if the earlier animal died) (Table I). Doses administered were as per recommendation of the AOT425StatPgm software. Animals were observed with special attention during the first $4 \mathrm{~h}$ and frequently thereafter, for a total of $48 \mathrm{~h}$. Masses of animals were recorded at the time of dosing and every $24 \mathrm{~h}$ thereafter. The mortality results of the animals were continuously fed into the AOT425StatPgm statistical program and the procedure was terminated after dosing six animals.

\section{In vivo antitumor efficacy testing}

The antitumor efficacy of 5-FU in solution and of thermoreversible C-GP-5FU gels was investigated in subcutaneously grown murine colon tumor model colon-26 in $\mathrm{BALB} / \mathrm{c}$ mice. Initially, a donor tumor-bearing BALB/c mouse was sacrificed and the tumor was cut into $2 \mathrm{~mm}^{3}$ pieces. Tumor pieces were transplanted in the dorsal hind limb area into 18 experimental male BALB/c mice each six to eight weeks of age and weighing 18-22 g. The animals were housed in autoclaved and secured cages throughout the study.

The tumor was allowed to grow for a week after which the animals were divided randomly into three groups, with six mice each. In case of treated animals, a calculated volume of injection was administered intratumorally using a 22 gauge needle. Each treated animal received a dose equivalent to $60 \mathrm{mg} \mathrm{kg}^{-1}$ of 5-FU. The first group received the test formulation (C-GP-5FU), second group was treated with the commercial formulation (Fivoflu ${ }^{\circledR} 250$, Dabur $50 \mathrm{mg} \mathrm{mL}^{-1}$ ) and the third group served as the untreated control. Animals were dosed starting on day 1 and then after skipping three days for a total of 17 days (5 injections). Following a schedule similar to that of drug administration, tumor volume and masses of animals as well as animal mortality were recorded for a period of 32 days. These measurements were continued for 15 days after the last injection on day 17.

Tumor volume was measured by Max-cal calipers. The relative tumor volume $(R T V)$ was expressed as $V / V_{0}$ index $(12,13)$ where

$V=$ Tumor volume on the last day of measurement

$V_{0}=$ Initial volume of the same tumor on the day the measurement was started.

Mortality of the animals was also recorded. The data obtained was analyzed statistically using one-way ANOVA Bonferroni's multiple comparison test $(p<0.01)$.

\section{RESULTS AND DISCUSSION}

\section{Preparation and evaluation of gels}

Gradual addition of GP solution to chitosan at temperatures lower than $15{ }^{\circ} \mathrm{C}$ was found to be crucial for the preparation of thermoreversible systems. The prepared sols were found to set into gels at physiological temperature after 8-10 min. The nearly trans- 
B. P. Depani et al:: Preparation and evaluation of chitosan based thermoreversible gels for intraperitoneal delivery of 5-fluorouracil (5-FU), Acta Pharm. 63 (2013) 479-491.

parent gels gradually turned opaque with the increase in temperature. Gel appearance at different temperatures $\left(15,25,37\right.$ and $\left.40^{\circ} \mathrm{C}\right)$ can be seen in Fig. 1 . On inverting, the formulation was flowable at all temperatures tested until physiological temperature was attained. At $37^{\circ} \mathrm{C}$, the sol was found to set into a relatively stiff mass that did not flow upon tube inversion. The $\mathrm{pH}$ of all solutions and gel formulations was found to be almost neutral.

Chitosan is a cationic polysaccharide soluble in aqueous media of $\mathrm{pH}<6.2$. In the absence of GP, repulsion between the positively charged chitosan chains did not allow any interactions between the chains. However, GP reduced the positive charge density on chitosan chains permitting hydrophobic attraction and hydrogen bonding between chitosan chains. Electrostatic attraction between the phosphate groups of the GP molecule and the ammonium groups of chitosan contributed further to gelation. The rate and manner of GP addition was found to be critical for formation of the desired gel. Addition of GP at a rapid rate or at higher temperature was found to result in chitosan precipitation. Rapid addition of GP into the chitosan solution resulted in a rapid increase in $\mathrm{pH}$ without allowing for any of the above occurrences and hence resulted in chitosan precipitation. The number of charged ammonium groups on the chitosan chain has been reported to be an important parameter controlling gelation in this system $(14,15)$.

Thermoreversibility of chitosan/GP systems is attributed to reduced chitosan chain polarity and increased hydrophobicity, increased interchain hydrophobic attraction and thermally induced transfer of protons from chitosan amine groups to the phosphate moiety of $\beta$-GP, leading to an attractive interchain interaction between chitosan chains. Unlike hydrogen bonds, hydrophobic forces are known to be temperature dependent and are suggested to be the source of thermoreversibility found in C-GP gels.

The in vitro release studies revealed that after an initial burst, 5-FU was released in a sustained manner from the gels (Fig. 3) and only about $72 \%$ of entrapped drug was released at the end of $12 \mathrm{~h}$, indicating prolonged release of 5-FU. Also, at the end of $12 \mathrm{~h}$, the gel was found to remain intact within the release chamber. On exposure to release medium, gels released as a burst about $40 \%$ of entrapped drug during the first hour, possibly due to surface 5-FU or due to the drug distributed in the tunnel of the gel during the gelation process, which diffuses rapidly when the gel comes into contact with the release medium. Following the initial burst, the 5-FU entrapped into the hydrogel was released gradually over the $12 \mathrm{~h}$ period of measurement. Burst release in the case of

Fig. 3. In vitro release of 5-FU from C-GP-5FU gel.

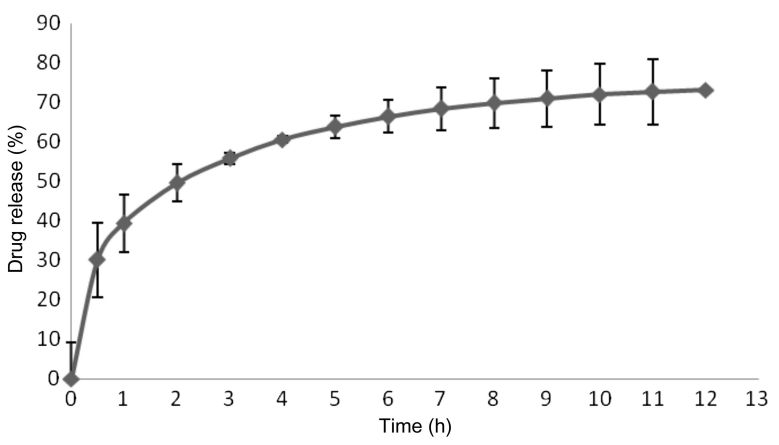


5-FU could be beneficial in attaining high drug levels in the tumor immediately after administration of the formulation. Gradual release that follows should serve to maintain the drug level at the site of action for a prolonged period. Studies have shown that C-GP systems can release the entrapped compound over a period of several hours to days depending upon the molecular weight of chitosan used and also that the phenomena of burst release of the entrapped compound is observed initially, followed by gradual release, irrespective of molecular mass of the entrapped drug. Pilocarpine entrapped in C-GP containing systems for ocular delivery showed fast release of the entrapped drug initially, after which the drug was released gradually over a period of $24 \mathrm{~h}$. A similar trend was observed for compounds like chlorpheniramine, calcein, etc. (9).

\section{Acute toxicity studies: Determination of $\mathrm{LD}_{50}$}

During the studies used for $L D_{50}$ determination, masses of animals dosed at all levels were found to decrease as the time proceeded (Table I). All three animals dosed at the highest dose of $550 \mathrm{mg} \mathrm{kg}^{-1}$ died whereas those dosed at the lower level of $175 \mathrm{mg} \mathrm{kg}^{-1}$ survived. Based on these results, the $L D_{50}$ of the test formulation was calculated to be $285 \mathrm{mg} \mathrm{kg}^{-1}$ as per the AOT425StatPgm. The $L D_{50}$ of 5 -FU on intraperitoneal administration to mice is reported to be $100 \mathrm{mg} \mathrm{kg}^{-1}$ (16). Thus, a 2.8 -fold decrease in toxicity of the test compound was observed.

The test drug in the present studies has a narrow therapeutic index (17). Following intraperitoneal administration of 5-FU solution to human subjects, the drug concentration was four times greater in portal venous blood compared to that sampled from a peripheral artery or vein or from the hepatic vein (18). Furthermore, up to $70 \%$ of the drug was extracted by the liver during the first pass. Seven to $20 \%$ of the parent drug was excreted unchanged in the urine in 6 hours; over $90 \%$ of this was excreted during the first hour. The remaining percentage of the administered dose was metabolized, primarily in the liver. The catabolic metabolism of fluorouracil resulted in degradation products (e.g., $\mathrm{CO}_{2}$, urea and $\alpha$-fluoro- $\beta$-alanine), which were inactive (19).

In the present study, a decrease in toxicity in terms of reduced mortality and absence of any significant change in the mass of dosed animals was observed compared to conventional injection. This decrease in toxicity can be attributed to a gradual and localized presentation of the cytotoxic agent from the formulation at the site of administration, which reduced systemic exposure and hence side effects. This is supported by earlier findings during phase I clinical studies on intraperitoneal formulations of 5-FU, which revealed that the concentration of the drug in the peritoneal cavity was 300 times greater than that in the plasma (20).

\section{In vitro cell line cytotoxicity studies}

Cytotoxicity of the drug free C-GP systems was found to be concentration dependent when evaluated in vitro in L-929 mouse fibroblast cells by an MTT assay. There was no significant difference between the percent proliferation of controls and those of test samples containing 10 to $50 \mu \mathrm{L}$ of C-GP gels $(p<0.05)$, indicating the absence of cytotoxicity. But the test wells containing $100 \mu \mathrm{L}$ of the C-GP system showed a significant difference in percent proliferation of fibroblast cells in comparison with the control $(p<$ 0.05) and exhibited toxic effects indicated by lower percent proliferation (60.57\%) (Fig. 4). 
Fig. 4. Effect of addition of different volumes of the C-GP system per well on percent proliferation of L-929 mouse fibroblast cells $24 \mathrm{~h}$ after co-incubation.

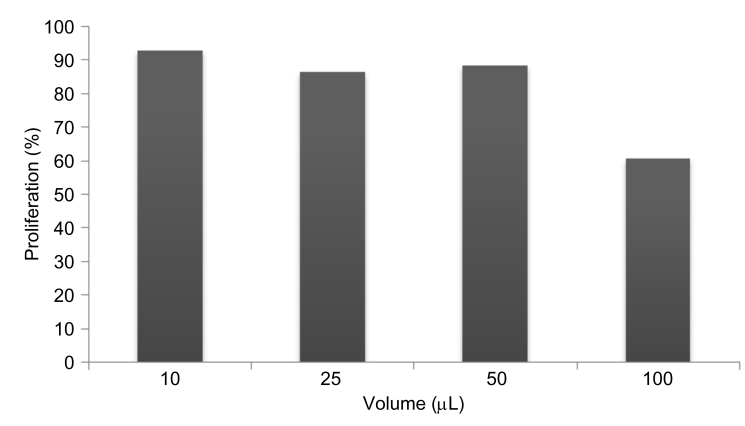

Chitosan is recognized as a biocompatible polymer (21) with GRAS status. However, the C-GP system used in the present studies is relatively unexplored as regards its toxicity. Since the system was intended to be used as an injectable depot, bioreactivity of drug free gels was investigated on mammalian L-929 fibroblast cells with the help of an MTT assay. L-929 mouse fibroblast cells have been used for evaluation of toxicity of newly synthesized polymeric materials and for evaluation of biocompatibility of novel drug delivery systems (22). Percent proliferation of fibroblast cells in contact with different volumes of the chitoan-GP systems is indicative of cell viability.

In an earlier report, C-GP systems containing $1 \%(m / V)$ chitosan and GP concentrations ranging from 5-20\% $(\mathrm{m} / \mathrm{V})$ were prepared and cytotoxicity of the gels was monitored using an extraction test by measuring the proliferation of goat bone marrow derived stem cells (gMSCs). Cytotoxicity of C-GP gels was found to increase with increasing GP concentrations, but extraction fluids from C-GP with $10 \%$ GP concentrations enhanced the proliferation of gMSCs 4 to 11-fold compared to the negative control. It was also found that chitosan with low GP concentrations $(5-10 \%, m / V)$ was not cytotoxic but enhanced the growth and proliferation of gMSCs (23).

The present study revealed a concentration dependent toxicity of the C-GP-system for L-929 mouse fibroblast cells. While addition of lower amounts of C-GP systems to the wells had no adverse effect on cell proliferation, the wells with higher amount (100 $\mu \mathrm{L}$ ) of gel showed a statistically significant reduction in cell proliferation. This could be attributed to the toxicity exerted by the system under evaluation at high concentrations, and/or a two-fold dilution of DMEM in these wells, and/or the presence of rigid gels in the wells when incubated at $37^{\circ} \mathrm{C}$, which could result in exertion of mechanical pressure on the underlying cells. Nevertheless, the results indicate the need to exercise caution in the in vivo use of these thermoreversibly gelling systems.

\section{In vivo anti-tumor efficacy testing}

All the control animals showed steady progression in the growth of the murine colon tumor model colon-26. However, their body weights and survival were not adversely affected by this growth and no morbidity and mortality were evident. A statistically significant difference in the mass of animals and mortality was observed in the 5-FU and C-GP-5-FU treated groups. At the dose administered, 5-FU solution injected intratumorally demonstrated toxicity in terms of mass loss and mortality. However, no significant 
B. P. Depani et al.: Preparation and evaluation of chitosan based thermoreversible gels for intraperitoneal delivery of 5-fluorouracil (5-FU), Acta Pharm. 63 (2013) 479-491.

mass loss was observed and no mortality was seen in the C-GP-5-FU gel treated mice up to day 32 although the dose administered remained identical. Tumor volume measurements indicate good efficacy when the results were analyzed using two-way ANOVA Bonferroni's multiple comparison test $(p<0.001)$. After a period of 17 days of treatment, the increase in tumor volume of the control was significantly higher than that in both treated groups (Figs. $5 \mathrm{a}$ and $5 \mathrm{~b}$ ). The tumor volume in control mice increased from 0.09 $\pm 0.03 \mathrm{~cm}^{3}(n=6)$ on day 1 of the study to $4.67 \pm 0.84 \mathrm{~cm}^{3}$ on day 32 . In contrast, tumor volumes in the chitosan-GP-5FU treated mice, which were comparable on day $1(0.087 \pm$ $0.016)$, were measured as $0.87 \pm 0.78 \mathrm{~cm}^{3}$ after 32 days. However, the given dose of 5 -FU as a commercial formulation was not tolerated by the 5-FU treated group, and animals, although with regressed tumors, showed severe mortality beyond 10 days of treatment and none of the animals survived beyond 17 days (Fig. 6a). In contrast, almost all the animals treated with chitosan gel survived throughout the experimental period. Reduced toxicity was also evident from the masses of animals (Fig. 6b). Whereas the animals treated with the 5-FU solution lost body mass soon after the treatment, no mass loss was observed in animals treated with C-GP-5-FU during the experimental period. The relative tumor volume was found to be lower for mice treated with both 5-FU solution and C-GP-5-FU containing gel, since the tumor volume remained constant until the end of the $5^{\text {th }}$ injection. After 17 days, the tumor volume in treated groups was found to be increase due to discontinuation of injections (Fig. 6c). The animals treated with 5-FU in solution did not survive beyond 17 days.

Since 5-FU is widely used in colonic cancers, in the present study subcutaneously grown murine colon tumors were used for evaluation of the developed formulation. Although the proposed route of delivery is intraperitoneal, as a limitation of our experiment, the colonic tumors could only be grown subcutaneously. Hence, injections were administered intratumorally. Since injections were given intratumorally, localization of the drug in the tumor would lead to reduced toxicity. To determine the therapeutic outcome of formulations after intratumoral injection, the rate of tumor progression over time was measured following multiple injections of 5-FU in solution as well as C-GP-5FU. Control mice showed rapid progression of tumor size and tumor volumes were significantly higher than those of the animals receiving intratumoral injections of a cytotoxic agent. High levels of mortality and morbidity seen in the group treated with 5-FU solu-
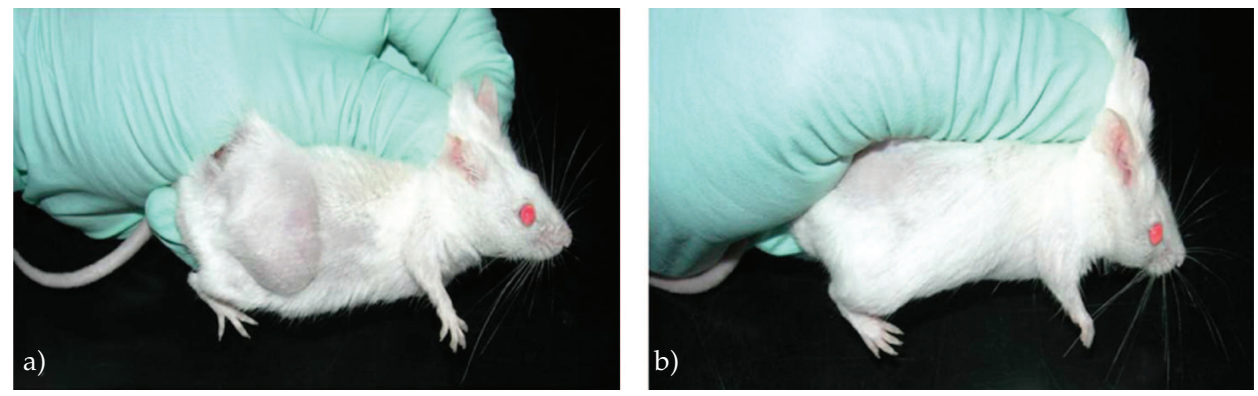

Fig. 5. a) Tumor in untreated control BALB/c, b) tumor in C-GP-5-FU gels treated BALB/c at the end of study. 
B. P. Depani et al.: Preparation and evaluation of chitosan based thermoreversible gels for intraperitoneal delivery of 5-fluorouracil (5-FU), Acta Pharm. 63 (2013) 479-491.
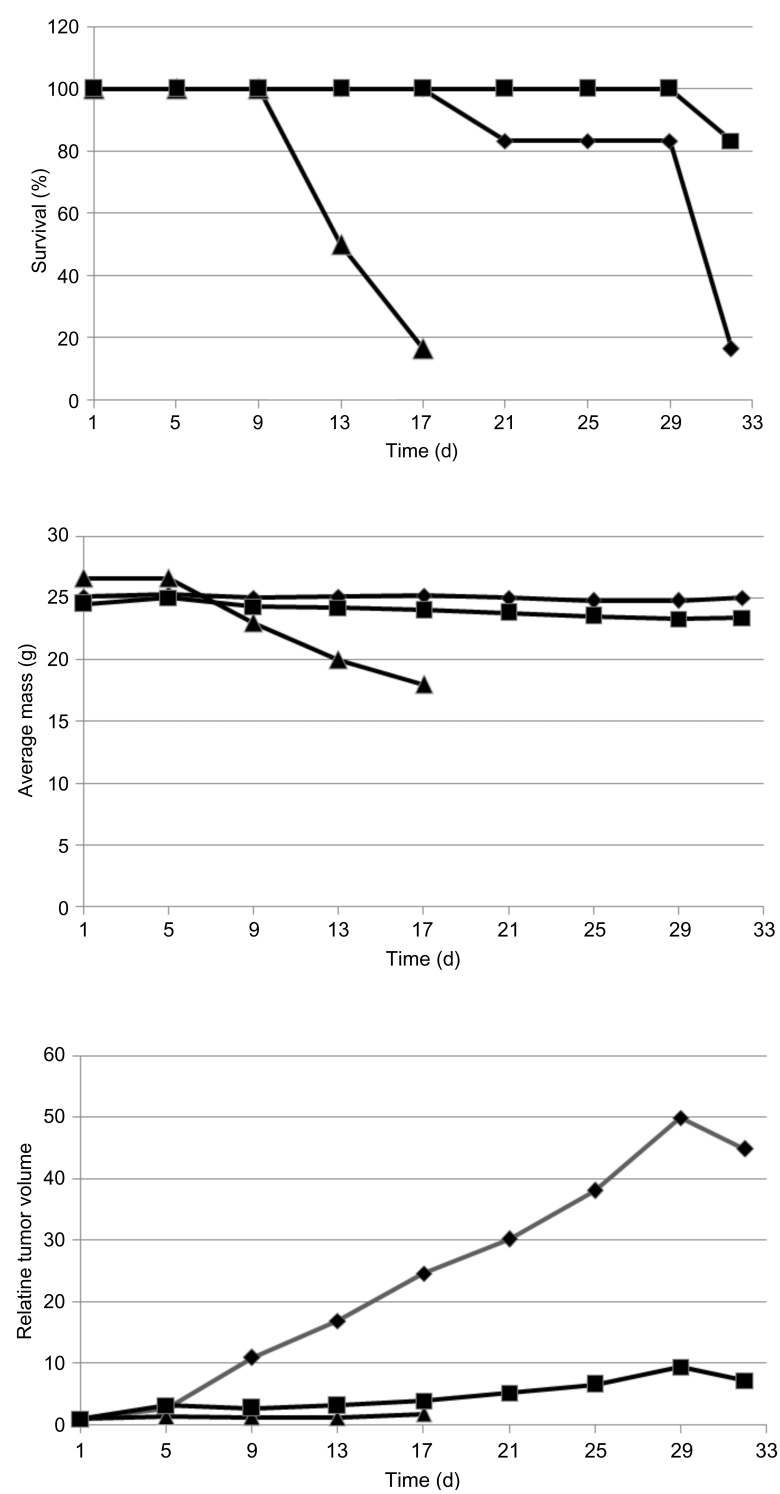

Fig. 6. a) Percent survival, b) body mass and c) relative tumor volume estimated in the control $(\bullet)$, C-GP-5FU treated $(\boldsymbol{\bullet})$ and 5 -FU treated BALB/c $(\boldsymbol{\Delta})(n=6$ per group). (Treated groups were administered a formulation equivalent to $60 \mathrm{mg} \mathrm{kg}^{-1}$ 5-FU intratumorally, once every 3 days).

tion provided evidence that the drug redistributes from tumors to result in systemic toxicity. In contrast, animals belonging to the C-GP-5FU treated group remained relatively free of morbidity and survived the entire test period. The relative tumor volume for both 
B. P. Depani et al.: Preparation and evaluation of chitosan based thermoreversible gels for intraperitoneal delivery of 5-fluorouracil (5-FU), Acta Pharm. 63 (2013) 479-491.

5-FU and C-GP-5FU treated mice remained close to one and any difference found was statistically insignificant $(p<0.01)$, indicating comparable efficacy. In case of the 5-FU solution treated mice, tumor volume was not measured beyond day 17 since animals did not survive. After the $17^{\text {th }}$ day of injection schedule, however, the relative tumor volume gradually increased, i.e., tumor progression was noted. This was attributed to exhaustion of the administered drug since no injections were given after day 17.

Lower morbidity and mortality associated with animals of the C-GP-5FU treated group is a clear indicator of its lower systemic toxicity. The substantially lower systemic exposure derived from gradual release of the drug into the tumor, followed by its uptake into tumor cells, resulted in localization of the drug in the tumor site. The low but sustained drug levels prevented tumor progression, but did not provide a sufficient driving force for diffusion of the drug into systemic circulation. This could be the reason for reduced toxicity of the developed systems. Thus, comparable efficacy adjudged from tumor volume measurements coupled with lower morbidity and mortality indicates that the developed delivery system could be a promising agent for the treatment of cancers of peritoneal organs.

Thermoreversible systems based on C-GP demonstrate a significant reduction in toxicity while retaining the efficacy of the cytotoxic drug 5-FU, as seen from the present studies. The C-GP system investigated here needs further efficacy and toxicity evaluation when administered intraperitoneally. Further, the system can be envisaged for use in drug delivery via a number of routes, including parenteral (subcutaneous, intramuscular and intraperitoneal) and nasal, to achieve prolonged drug residence at these sites.

Acknowledgement. - The authors gratefully acknowledge the support of Naprod Life Sciences for 5-FU, BARC for radiation sterilization of 5-FU, Central Institute of Fisheries Technology, India, for chitosan sample and ACTREC, Mumbai, India, for helping with the in vivo efficacy studies.

\section{REFERENCES}

1. S. C. Sweetman, Martindale the Complete Drug Reference (Ed. S. C. Sweetman), $34^{\text {th }}$ ed., Pharmaceutical Press, London 2005, pp. 554-556.

2. E. Reed, J. Jacob, R. F. Ozols, R. C. Young and C. Allegra, 5-Fluorouracil (5-FU) and leucovorin in platinum-refractory advanced stage ovarian carcinoma, Gynecol. Oncol. 46 (1992) 326-329; DOI: 10.1016/0090-8258(92)90226-9.

3. www.rxlist.com, accessed on $25^{\text {th }}$ July 2011.

4. A. Goodman and L. Gilman, Antineoplastic Agents, in Manual of Pharmacology and Therapeutics (Ed. K. Parker), 11 ${ }^{\text {th }}$ ed., McGraw Hill, New York 2008, pp. 874-876.

5. M. Markman, Intraperitoneal antineoplastic drug delivery: rationale and results, Lancet Oncol. 4 (2003) 277-283; DOI: 10.1016/S1470-2045(03)01074-X.

6. A. Chenite, M. Buschmann, D. Wang, C. Chaput and N. Kandani, Rheological characterisation of thermogelling chitosan/glycerol-phosphate solutions, Carbohydr. Polym. 46 (2001) 39-47; DOI: 10.1016/S0144-8617(00)00281-2.

7. W. Jie, S. Zhi-Guo and M. Guang-Hui, A thermo- and pH-sensitive hydrogel composed of quaternized chitosan/glycerophosphate, Int. J. Pharm. 315 (2006) 1-11; DOI: 10.1016/j.ijpharm.2006. 01.04 . 
B. P. Depani et al:: Preparation and evaluation of chitosan based thermoreversible gels for intraperitoneal delivery of 5-fluorouracil (5-FU), Acta Pharm. 63 (2013) 479-491.

8. Y. Wang, N. Xu, Q. Luo, Y. Li, L. Sun, H. Wang, K. Xu, B. Wang and Y. Zhen, In vivo assessment of chitosan/â-glycerophosphate as a new liquid embolic agent, Interv. Neuroradiol. 17 (2011) 87-92.

9. E. Ruel-Gariepy and L. Jean-Christophe, In situ-forming hydrogels-review of temperature-sensitive systems, Eur. J. Pharm. Biopharm. 58 (2004) 409-426; DOI: 10.1016/j.ejpb.2004.03.019.

10. E. Ruel-Gariepy, A. Chenite, C. Chaput, S. Guirguis and J. Leroux, Characterization of thermosensitive chitosan gels for the sustained delivery of drugs, Int. J. Pharm. 203 (2000) 89-98; DOI: 10.1016/S0378-5173(00)00428-2.

11. OECD guideline 425 for the testing of chemicals. Section 4, Acute Oral Toxicity: Up-and-Down Procedure.

12. G. Carlsson, B. Gullberg and L. Hafstrom, Estimation of liver tumor volume using different formulas - an experimental study in rats, J. Cancer Res. Clin. Oncol. 105 (1983) 20-23; DOI: 10.1007/ BF00391826.

13. M. M. Tomayko and C. P. Reynolds, Determination of subcutaneous tumor size in athymic (nude) mice, Cancer Chemother. Pharmacol. 24 (1989) 148-154; DOI: 10.1007/BF00300234.

14. B. Jeong, S. W. Kim and Y. H. Bae, Thermosensitive sol-gel reversible hydrogels, Adv. Drug Deliv. Rev. 54 (2002) 37-51; DOI: 10.1016/S0169-409X(01)00242-3.

15. F. Ganji, M Abdekhodaie and S. A. Ramazani, Gelation time and degradation rate of chitosan-based injectable hydrogel, J. Sol-Gel Sci. Tech. 42 (2007) 47-53; DOI: 10.1007/s10971-006-9007-1.

16. D. Anderson and D. M. Conning, Experimental Toxicology: the Basic Issues, $2^{\text {nd }}$ ed., Royal Society of Chemistry, California 1993, p. 17.

17. A. M. Fan, L. W. Chang, Toxicology and Risk Assessment; Principles, Methods and Application, $4^{\text {th }}$ ed., Marcel Dekker Inc., New York 1996, pp. 108-110.

18. J. L. Speyer, P. H. Sugarbaker, J. M. Collins, R. L. Dedrick, R. W. Klecker and C. E. Myers, Portal levels and hepatic clearance of 5-fluorouracil after intraperitoneal administration in humans, Cancer Res. 41 (1981) 1916-1922.

19. M. J. Ellenhorn, Ellenhorn's Medicinal Toxicology; Diagnosis and Treatment of Human Poisoning (Ed. M. J. Ellenhorn), $2^{\text {nd }}$ ed., Williams and Wilkins, Baltimore 1997, p. 1326.

20. R. L. Schilsky, K. E. Choi, J. Grayhack, D. Grimmer, C. Guarnieri and L. Fullem, Phase I clinical and pharmacologic study of intraperitoneal cisplatin and fluorouracil in patients with advanced intra-abdominal cancer, J. Clin Oncol. 8 (1990) 2054-2061.

21. M. Ravi Kumar, R. Muzzarelli and C. Muzzarelli, Chitosan chemistry and pharmaceutical perspectives, Chem. Rev. 104 (2004) 6017-6084; DOI: 10.1002/chin.200511296.

22. R. Zange, Y. Li and T. Kissel, Biocompatibility testing of ABA triblock copolymers consisting of poly (L-lactic-co-glycolic acid) A blocks attached to a central poly(ethylene oxide) B block under in vitro conditions using different L929 mouse fibroblasts cell culture models, J. Control. Release 56 (1998) 249-258; DOI: 10.1016/S0168-3659(98)00093-5.

23. R. Ahmadi, M. Zhou, J. D. De Bruijn, The use of thermo-sensitive chitosan as an injectable carrier for bone tissue engineering, Eur. Cell Mater. 10 (2005) 61. 